\title{
Optimal Control Model of Human Three-Joint Arm System Characterized by Hand-Joint's Freezing-Like Mechanism*
}

\author{
Toshikazu MATSUI**, Kouki TAKESHITA** and Takahisa SHIBUSAWA** \\ ** Graduate School of Engineering, Gunma University, \\ 29-1 Hon-cho, Ota-shi, Gunma 373-0057, Japan \\ E-mail:matsui@gunma-u.ac.jp
}

\begin{abstract}
This paper proposes a new optimal control model of the human three-joint arm system (shoulder, elbow, and hand joints) and clarifies its basic performance. The proposed model is characterized by a freezing-like mechanism in its hand joint and optimizes its criterion function composed of three kinds of energy costs and a torque-change cost. The model's freezing-like mechanism is formulated as a feedback controller to produce feedback torque in the direction opposite to the hand-joint motion. Consequently, it was clarified that the minimization of the moment power of joints or that of the energy consumed by viscous resistance made it possible to simulate human three-joint arm's reaching movements including the hand-joint's freezing-like characteristics at the constant weight values regardless of target positions, whereas the torque-change minimization failed to do so. This result suggests that the energy minimization model with the freezing-like mechanism in its hand joint can be effective as a general and plausible model of the human three-joint arm control system and that the two kinds of energy can be involved in the trajectory planning for human three-joint arm's reaching movements.
\end{abstract}

Key words: Reaching Movement, Optimal Control, Three-Joint Arm, Hand Joint, Freezing-Like Mechanism, Energy Minimization, Torque-Change Minimization

\section{Introduction}

In human arm's two-point reaching movements, the hand tends to move along a roughly straight path with a bell-shaped tangential velocity profile ${ }^{(1),(2)}$. So far, three typical kinds of optimal control models have been proposed to simulate human arm's reaching movements: minimum jerk model, minimum torque-change model, and minimum energy model. The first is a kinematical model that predicts the optimal hand trajectory by minimizing the square of jerk (the rate of change of acceleration) of the hand point integrated over the entire movement ${ }^{(3)}$. The second and third are dynamical models that generate the optimal hand trajectory by minimizing the square of the rate of change of torque ${ }^{(4),(5)}$ and the consumed energy ${ }^{(6),(7)}$, respectively, integrated over the entire movement. However, the conventional models have no ability to reproduce the variability of end points of the hand paths in human arm's reaching movements because their end-point boundary conditions are strictly determined. As models to reproduce the end-point variability of hand paths as well as reaching movement characteristics, two kinds of models have been proposed. The first is the minimum variance model that determines the optimal hand trajectory so as to minimize the variance in the fluctuation of the end points over a given period after the reaching 
movement, assuming that the hand trajectory is generated by the motor command signal including a noise (signal-dependent noise) with the variance depending on the signal's mean level ${ }^{(8)}$. The second is the modified minimum torque-change model formulated by modifying the end-point boundary conditions of the conventional minimum torque-change model from fixed ones into free ones ${ }^{(9)}$. The conventional models mentioned above are two-joint arm models composed of shoulder and elbow joints. However, in order to develop a more advanced model capable of simulating human reaching movements including grasping ones, the human arm's control mechanism should be formulated at least as a three-joint arm model composed of shoulder, elbow, and hand joints. Moreover, although situations often arise where hands are actively involved in reaching movements, hands can fundamentally be regarded as an end-effector. Therefore, hand joints should be modeled from the standpoint different from shoulder and elbow joints.

From the above point of view, we formulate a new optimal control model of the human three-joint arm system composed of shoulder, elbow, and hand joints, and verify how the proposed model can reproduce human three-joint arm's reaching movement characteristics. Actually, the proposed model is realized by extending the previous modified minimum torque-change model to a three-joint model characterized by a freezing-like mechanism in its hand joint and incorporating three kinds of energy costs (moment power of joints, energy for sustaining the torque of joints, and energy consumed by viscous resistance) into the previous model's criterion function. The freezing-like mechanism installed in the model represents a phenomenon that prevents the hand-joint angle from changing during movements, and is apparently similar to the freezing mechanism ${ }^{(10) \sim(12)}$ where the stiffness of joints is strengthened by contracting the agonist and antagonist muscles together. We model the freezing-like mechanism as a feedback controller to produce feedback torque in the direction opposite to the hand-joint motion. Consequently, it was clarified that the minimization of the moment power of joints or that of the energy consumed by viscous resistance made it possible to simulate human three-joint arm's reaching movements including the hand-joint's freezing-like characteristics at the constant weight values, whereas the minimization of the square of the torque-change failed to do so. This suggests that the energy minimization model with the freezing-like mechanism in its hand joint can be effective as a general and plausible model of the human three-joint arm control system and that the two kinds of energy can be involved in the trajectory planning for human three-joint arm's reaching movements. Section 2 verifies the existence of the hand-joint's freezing-like mechanism through measuring three-joint arm's reaching movements, and formulates a new human three-joint arm's optimal control model characterized by its hand-joint's freezinglike mechanism. In Section 3, we clarify the model's performance in simulating human three-joint arm's reaching movements. In Section 4, we discuss differences in performance between the proposed three-joint arm model and the conventional two-joint arm models such as the minimum torque-change and minimum energy models.

\section{Human Three-Joint Arm's Optimal Control Model}

\subsection{Experimental measurement of human three-joint arm's reaching movements}

Figure 1 shows an apparatus for measuring horizontally planar reaching movements. In the experiment, the subjects seated and held a hand-held bar $\left(m_{L}=1.2 \mathrm{~kg}\right)$ fixed at the end of a two-link mechanical manipulator with their fingertips, and performed their three-joint arm's horizontal two-point reaching movements toward the green LED targets mounted in the desk covered with a transparent Plexiglas sheet at the shoulder level. Figure 2 shows the arrangement of the start positions (S1, S2) and the target positions (T4, T5, T6, T7, T8). A red LED was mounted on each of the three joints and the hand-held bar. The hand paths from the start positions to the target positions were derived by recording the motion of the red LEDs with a digital video camera (30 frames/sec) placed above the experimental appa- 


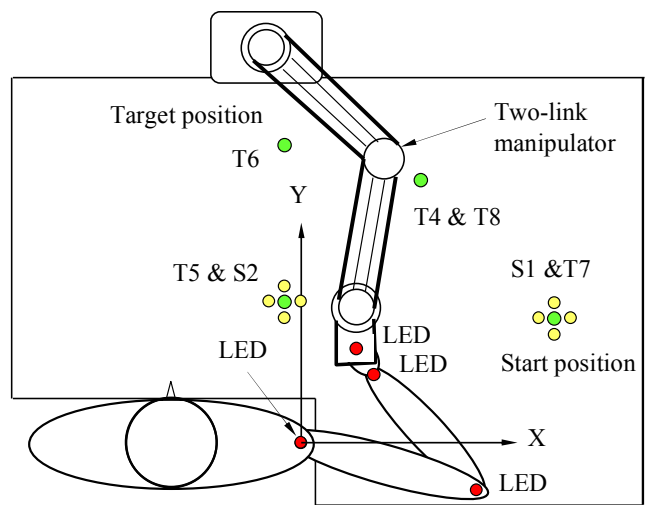

Fig.1 Experimental setup for measuring reaching movements

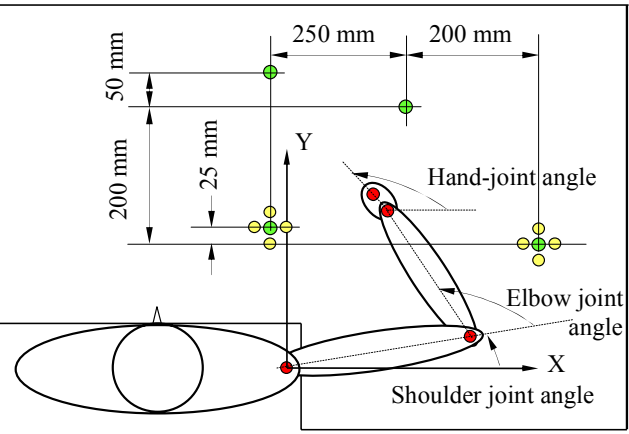

Fig.2 Arrangement of the start and target LEDs

ratus and analyzing it. The shoulder joint was set as the origin of the $\mathrm{X}-\mathrm{Y}$ coordinates.

The subjects were instructed just to perform two-point reaching movements toward the left or right direction: as for leftward reaching movements, the hand moved leftward from the start position S1 (the same position as T7) to one of the target positions T4, T5, and T6; as for rightward reaching movements, the hand moved rightward from the start position S2 (the same position as T5) to one of the target positions T7 and T8. There were not any other instructions. The experiment was executed exactly according to the following procedure.

(1) The subjects move the hand-held bar to the start LED (S1 or S2) while it is illuminated.

(2) All the target LEDs are illuminated for two seconds, and then they are turned off for a randomly changed duration between 1 and $2 \mathrm{sec}$.

(3) As soon as one randomly selected target LED is lit, the subjects move the hand-held bar from the start position to the target position.

Three subjects (T.S., K.T., and T.I.) participated in the experiment. Since 30 trials were performed for each target, a total of 90 trials and 60 trials were executed as the leftward and rightward movements, respectively. The experiment was conducted in a dark room. Off-line digital image signal processing was performed to compute the subjects' hand paths and hand-joint angles, and the shoulder- and elbow-joint angles were derived from the hand paths and the hand-joint angles based on the inverse kinematics. To multiply the number of measured data, the joint angles every $1 / 30 \mathrm{sec}$ were interpolated into those every $1 \mathrm{msec}$ using a least-squared method under the condition that the joint angles, their angular velocities, and their angular accelerations agreed with the measured ones at the start and final positions, and then the interpolated joint angles were differentiated to calculate their angular velocities and differentiated again to calculate their angular accelerations.

Figure 3 shows the shoulder-, elbow-, and hand-joint angle profiles derived from the measured reaching movements; (a) and (b) are for the leftward movements from S1 to T4 and T5, respectively; (c) is for the rightward movements from S2 to T8; (1), (2), and (3) are for subjects T.S., K.T., and T.I., respectively; 10-trial measured data overlap each other. As seen from Fig. 3, the shoulder- and elbow-joint angles changed definitely during the movement duration, whereas the hand-joint angle hardly changed over the movement duration though it was not always constant, and this result was independent of the start and target positions and the subjects. This gives us two implications. One is that human hand joints are hardly involved in human three-joint arm's reaching movements. This is because the phenomenon arises which is apparently similar to the freezing phenomenon ${ }^{(10)(12)}$ in which the stiffness of joints is strengthened by contracting the agonist and antagonist muscles together and because the human three-joint arm is moved as if it were a two-joint arm with no hand joint. In terms of the outward similarity, the experimental fact can be regarded as a freezing-like phenomenon. The other is that the human three-joint arm system 
does not completely fix its hand-joint angle but can change it a little depending on the start and target positions, even if the freezing-like phenomenon happens to the hand joint. Therefore, three-joint arm's optimal control models should possess the ability to reproduce the above freezing-like characteristics.
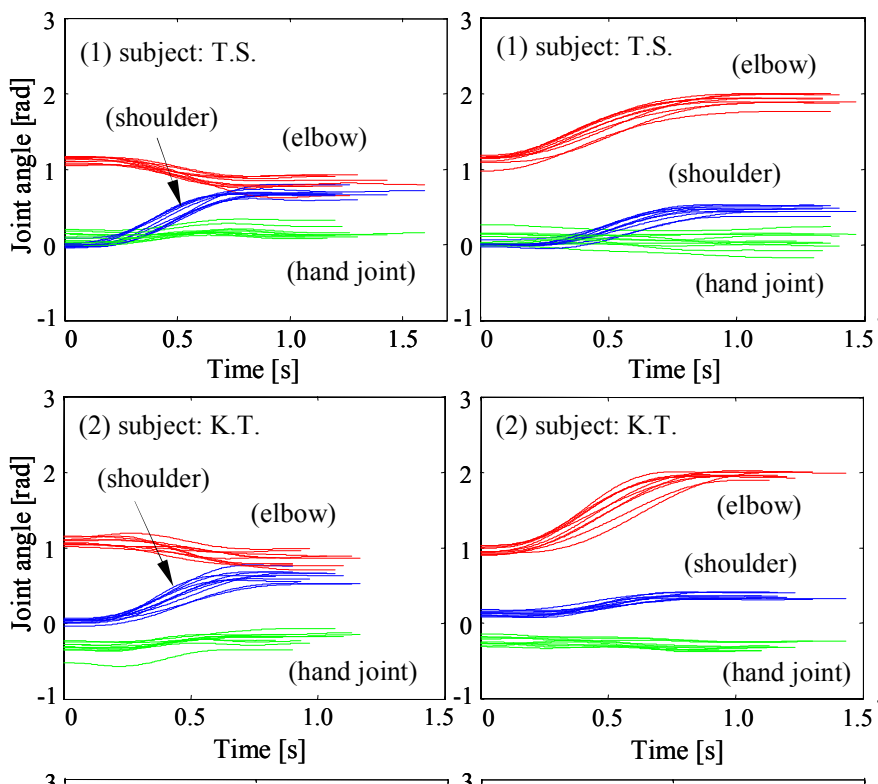

(1) subject: T.S.

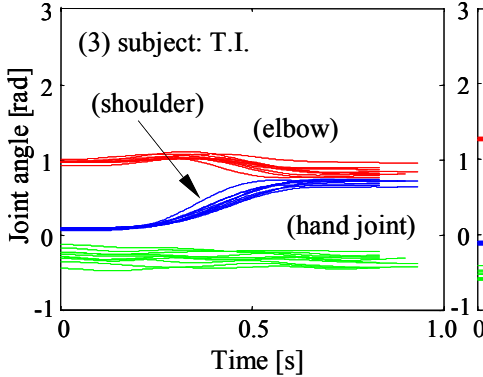

(2) subject: K.T.
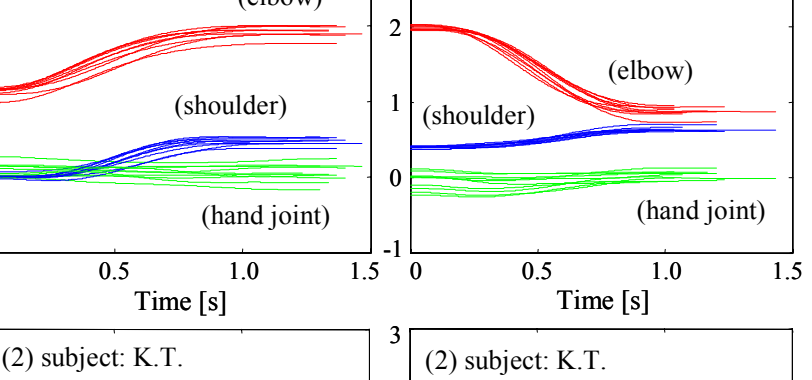

(2) subject: K.T.
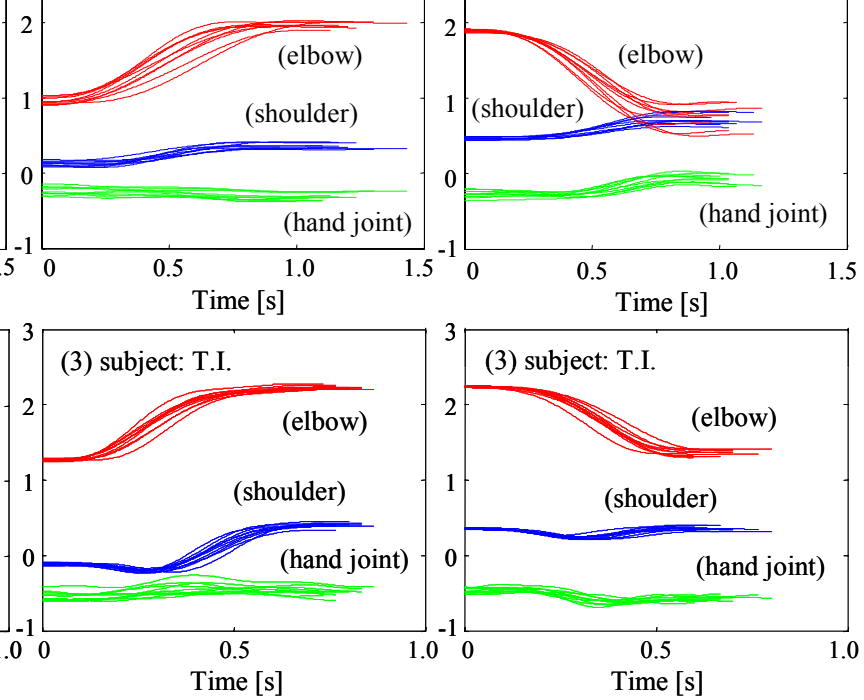

(3) subject: T.I.

(a) leftward movements (S1-T4)

(b) leftward movements (S1-T5)

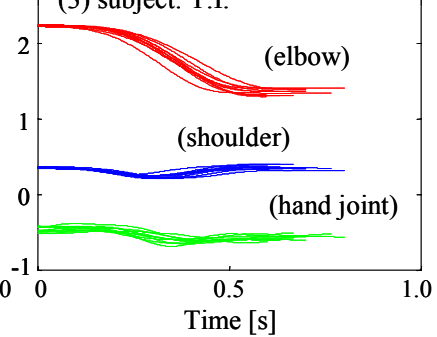

(c) rightward movements (S2-T8)

Fig. 3 Joint angle profiles for the measured three-joint arm's reaching movements

\subsection{Formulation of human three-joint arm's optimal control model}

As seen from Fig. 3, the hand-joint angle hardly changed over the movement duration for human three-joint arm's reaching movements. This can be thought to be caused by the freezing-like mechanism that prevents the hand-joint angle from changing. The hand-joint's freezing-like mechanism can be modeled as a feedback controller that produces feedback torque in the direction opposite to the hand-joint motion. Letting a desired hand-joint angle, its angular velocity, and its angular acceleration be all zero in the feedback controller, we can express the feedback torque $\tau_{3}{ }^{*}$ around the hand joint as follows:

$$
\tau_{3}{ }^{*}=-k_{a} \ddot{\theta}_{3}-k_{v} \dot{\theta}_{3}-k_{p} \theta_{3}
$$

where $k a, k v$, and $k p$ are the feedback gains. Therefore, if the human three-joint arm model illustrated in Figure 4 has the freezing-like mechanism in its hand joint, its equation of motion can be given as follows by adding the feedback torque $\tau_{3} *$ to the hand-joint's drive torque $\tau_{3}$ :

$$
\tau^{*}=\boldsymbol{M} \ddot{\boldsymbol{\theta}}+\boldsymbol{h}+\boldsymbol{D} \dot{\boldsymbol{\theta}}
$$

$$
\begin{aligned}
& \tau^{*}=\left[\tau_{1}, \tau_{2}, \tau_{3}+\tau_{3}{ }^{*}\right]^{\mathrm{T}}, \quad \theta=\left[\theta_{1}, \theta_{2}, \theta_{3}\right]^{\mathrm{T}} \\
& M_{11}=J_{1}+J_{2}+J_{3}+2 b_{12} \cos \left(\theta_{2}\right)+2 b_{13} \cos \left(\theta_{2}+\theta_{3}\right)+2 b_{23} \cos \left(\theta_{3}\right) \\
& M_{12}=M_{21}=J_{2}+J_{3}+b_{12} \cos \left(\theta_{2}\right)+b_{13} \cos \left(\theta_{2}+\theta_{3}\right)+2 b_{23} \cos \left(\theta_{3}\right) \\
& M_{13}=M_{31}=J_{3}+b_{13} \cos \left(\theta_{2}+\theta_{3}\right)+b_{23} \cos \left(\theta_{3}\right), M_{33}=J_{3} \\
& M_{22}=J_{2}+J_{3}+2 b_{23} \cos \left(\theta_{3}\right), M_{23}=M_{32}=J_{3}+b_{23} \cos \left(\theta_{3}\right)
\end{aligned}
$$




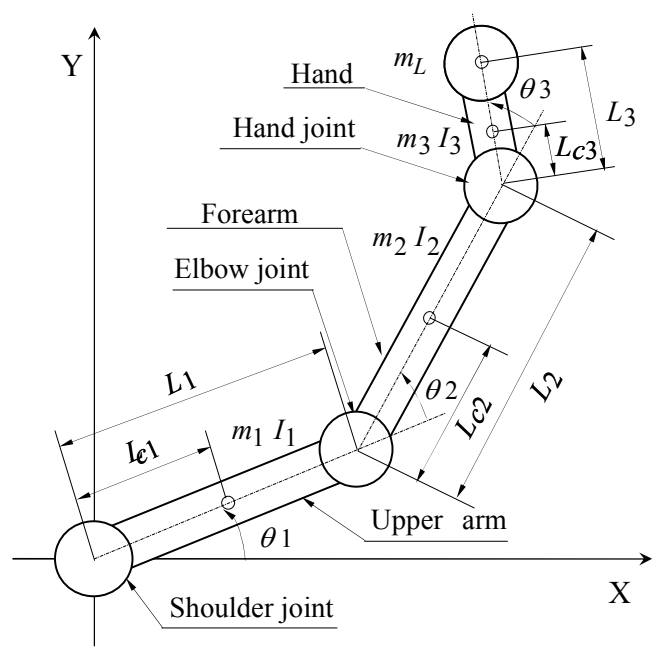

Table 1 Dynamics parameters of the human three-joint arm for one subject

Fig.4 A model of the human three-joint arm.

$$
\begin{aligned}
& J_{1}=m_{1} L_{c 1}{ }^{2}+\left(m_{2}+m_{3}+m_{L}\right) L_{1}{ }^{2}+I_{1} \\
& J_{2}=m_{2} L_{c 2}{ }^{2}+\left(m_{3}+m_{L}\right) L_{2}^{2}+I_{2}, J_{3}=m_{3} L_{c 3}{ }^{2}+m_{L} L_{3}{ }^{2}+I_{3} \\
& b_{12}=m_{2} L_{1} L_{c 2}+\left(m_{3}+m_{L}\right) L_{1} L_{2}, b_{13}=m_{3} L_{1} L_{c 3}+m_{L} L_{1} L_{3}, b_{23}=m_{3} L_{2} L_{c 3}+m_{L} L_{2} L_{3} \\
& h_{1}=\left\{-b_{12} \sin \left(\theta_{2}\right)-b_{13} \sin \left(\theta_{2}+\theta_{3}\right)\right\}\left(\dot{\theta}_{2}^{2}+2 \dot{\theta}_{1} \dot{\theta}_{2}\right)-\left\{b_{13} \sin \left(\theta_{2}+\theta_{3}\right)+b_{23} \sin \left(\theta_{3}\right)\right\}\left(\dot{\theta}_{3}{ }^{2}+2 \dot{\theta}_{2} \dot{\theta}_{3}+2 \dot{\theta}_{1} \dot{\theta}_{3}\right) \\
& h_{2}=\left\{b_{12} \sin \left(\theta_{2}\right)+b_{13} \sin \left(\theta_{2}+\theta_{3}\right)\right\} \dot{\theta}_{1}^{2}-b_{23} \sin \left(\theta_{3}\right)\left(\dot{\theta}_{3}{ }^{2}+2 \dot{\theta}_{2} \dot{\theta}_{3}+2 \dot{\theta}_{1} \dot{\theta}_{3}\right) \\
& h_{3}=\left\{b_{13} \sin \left(\theta_{2}+\theta_{3}\right)+b_{23} \sin \left(\theta_{3}\right)\right\} \dot{\theta}_{1}^{2}+b_{23} \sin \left(\theta_{3}\right)\left(\dot{\theta}_{2}{ }^{2}+2 \dot{\theta}_{1} \dot{\theta}_{2}\right)
\end{aligned}
$$

where $\theta_{i}, \dot{\theta}_{i}, \ddot{\theta}_{i}$, and $\tau_{i}$ denote the joint angle, the joint angular velocity, the joint angular acceleration, and the drive torque of the $i$-th joint, respectively. Links 1, 2, and 3 correspond to the upper arm, the forearm, and the hand, respectively, and joints 1, 2, and 3 correspond to the shoulder, elbow, and hand joints, respectively. The shoulder is the origin of the X-Y coordinates. $\boldsymbol{M}=[M i j](i, j=1 \sim 3), \boldsymbol{h}=\left[h_{1}, h_{2}, h_{3}\right]^{\mathrm{T}}$ and $\boldsymbol{D}$ represent the inertia matrix, the Coriolis and centrifugal force vector, and the $3 \times 3$ diagonal viscous matrix, respectively; superscript $\mathrm{T}$ denotes the transpose of a vector; $m_{i}$ and $m_{L}$ represent the mass of the $i$-th link and the mass $(=1.2 \mathrm{~kg}$ ) of the object (handle) held with the fingertips, respectively; $L i, L c i$, and $I i$ are the length of the $i$-th link, the length from the center of mass of the $i$-th link to the $i$-th joint, and the moment of inertia around the center of mass of the $i$-th link, respectively; $D i$ is the viscosity coefficient of the $i$-th joint. Since the human arm model shown in Fig. 4 moves within a horizontal plane at the height of the shoulder, the influence of gravity can be neglected. Table 1 summarizes the physical parameters of the arm for one subject. The values of $m i, L c i, I i$, and $D i(i=1 \sim 3)$ were estimated from the measured link length $L i$ for each subject by referring to references (5) and (7). Substituting Eq. (1) into Eq. (2) yields the following state equation:

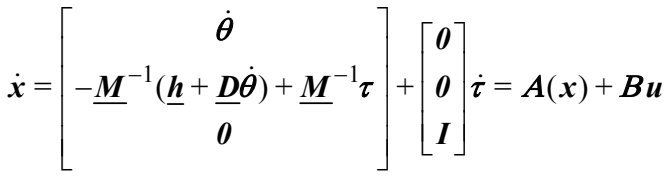

$$
\begin{aligned}
& \boldsymbol{x}=\left[\theta_{1}, \theta_{2}, \theta_{3}, \dot{\theta}_{1}, \dot{\theta}_{2}, \dot{\theta}_{3}, \tau_{1}, \tau_{2}, \tau_{3}\right]^{\mathrm{T}}, \boldsymbol{u}=\dot{\boldsymbol{\tau}}=\left[\dot{\tau}_{1}, \dot{\tau}_{2}, \dot{\tau}_{3}\right]^{\mathrm{T}}, \boldsymbol{I}: \text { unit matrix }(3 \times 3)
\end{aligned}
$$

where $\boldsymbol{x}$ and $\boldsymbol{u}$ represent the state variable vector and the input variable vector, i.e., the rate of change of torque; $\underline{\boldsymbol{M}}$ and $\underline{\boldsymbol{D}}$ are the matrices identical to $\boldsymbol{M}$ and $\boldsymbol{D}$, respectively, except that the $(3,3)$ components of $\boldsymbol{M}$ and $\boldsymbol{D}$ are replaced by $M_{33}+k a$ and $D_{3}+k v$, respectively; $\underline{\boldsymbol{h}}$ is the vector identical to $\boldsymbol{h}$ except that the third component is replaced by $h_{3}+k p \theta_{3}$. Therefore, incorporating the feedback mechanism into the model's hand joint is equivalent to increasing the $(3,3)$ components of $\boldsymbol{M}$ and $\boldsymbol{D}$ and the third component of $\boldsymbol{h}$ to make the hand joint difficult to move.

The conventional two-joint arm's optimal control models mainly minimize two kinds of 
criteria: the square of the rate of change of torque and the consumed energy integrated over the entire movement. As for the proposed three-joint arm's optimal control model, we combine the both criteria to discuss also which is more effective in simulating reaching movements. Actually, the proposed optimal control model minimizes the following criterion function subject to the constraint imposed by the state equation of Eq. (3) with its handjoint's freezing-like mechanism.

$$
\begin{aligned}
& J=\left\{\boldsymbol{x}\left(t_{f}\right)-\boldsymbol{x}_{t f}\right\}^{\mathrm{T}} \boldsymbol{Q}\left\{\boldsymbol{x}\left(t_{f}\right)-\boldsymbol{x}_{t f}\right\}+\int_{0}^{t_{f}}\left(W+\omega_{4} \boldsymbol{u}^{\mathrm{T}} \boldsymbol{R} \boldsymbol{u}\right) d t \\
& W=\omega_{1} \tau^{\mathrm{T}} \dot{\boldsymbol{\theta}}+\omega_{2} \tau^{\mathrm{T}} \tau+\omega_{3} \dot{\boldsymbol{\theta}}^{\mathrm{T}} \boldsymbol{D} \dot{\boldsymbol{\theta}}
\end{aligned}
$$

The first term of the criterion function (Eq. (4)) represents the error between the state variable at the final point and its desired value, and the second term of $J$ represents the weighted sum of three kinds of energy costs and the square of the torque-change integrated over the entire movement. The first, second, and third costs of the energy term $W$ (Eq. (5)) represent the moment power of joints, the energy for sustaining the torque of joints, and the energy consumed by viscous resistance, respectively, and $\omega_{1}, \omega_{2}, \omega_{3}$, and $\omega_{4}$ are the weight values. In Eq. (4), $t_{f}$ is the movement duration; $\boldsymbol{x}\left(t_{f}\right)$ and $\boldsymbol{x}_{t f}$ are the value of the state variable $\boldsymbol{x}$ at the final point $\left(t=t_{f}\right)$ and its desired value, respectively; $\boldsymbol{Q}$ and $\boldsymbol{R}$ are the $9 \times 9$ and $3 \times 3$ diagonal weight matrices, respectively. Human arm's reaching movements are realized by the vision mechanism finding the target position and then by the arm control mechanism moving the arm. Therefore, at least two problems must be solved to formulate optimal control models that reproduce measured reaching movement characteristics. The first is which should be incorporated into such models as the target information, task coordinates of the target acquired by the vision mechanism or their body coordinates (joint angles); this relates closely to the first term of Eq. (4). The second is what optimization criterion is necessary for such models to reproduce measured reaching movement characteristics; this relates closely to the second term of Eq. (4). In this paper, the measured joint angles at the final point are substituted into the first term of Eq. (4) to avoid the first problem. This is based on the idea that the highest priority is to solve the second problem, i.e., to clarify what criterion makes it possible to reproduce measured reaching movement characteristics, because there has been no research on three-joint arm's optimal control models composed of shoulder, elbow, and hand joints. The optimization of Eq. (4) can be solved by using the calculus of variations in the same way as that of ordinary optimal control problems. Let a Hamiltonian be defined as

$$
H(\boldsymbol{x}, \boldsymbol{u}, \lambda)=W+\omega_{4} \boldsymbol{u}^{\mathrm{T}} \boldsymbol{R} \boldsymbol{u}+\lambda^{\mathrm{T}}(\boldsymbol{A}(\boldsymbol{x})+\boldsymbol{B} \boldsymbol{u}) .
$$

Then, the control which optimizes the criterion function of Eq. (4) can be derived from the following equation.

$$
\partial H / \partial \boldsymbol{u}=\boldsymbol{0} .
$$

The optimal trajectory can be determined by solving the following set of nonlinear differential equations with respect to $\boldsymbol{x}$ and $\boldsymbol{\lambda}$, which is a necessary condition for an optimum point to exist, under the optimal control of Eq. (7).

$$
\boldsymbol{x}=\partial H / \partial \lambda, \quad \boldsymbol{\lambda}=-\partial H / \partial \boldsymbol{x}
$$

where $\lambda$ denotes the adjoint variable vector with 9 components. Therefore, Eq. (8) can be solved as a two-point boundary-value problem under the following boundary conditions.

$$
\boldsymbol{x}(0)=\boldsymbol{x}_{0}, \quad \lambda\left(t_{f}\right)=2 \boldsymbol{Q}\left\{\boldsymbol{x}\left(t_{f}\right)-\boldsymbol{x}_{t f}\right\},
$$

where $\boldsymbol{x}_{0}$ and $\lambda\left(t_{f}\right)$ are the initial value of the state variable $\boldsymbol{x}$ at the start point $(t=0)$ and the final value of the adjoint variable $\lambda$ at the final point $\left(t=t_{f}\right)$, respectively. This two-point boundary-value problem can be solved iteratively based on a Newton-like method.

\section{Model's Performance in Reproduction of Reaching Movements}

\subsection{Trajectory reproduction based on torque-change minimization criterion}

Figure 5 shows the influence of the weight matrix $\boldsymbol{R}$ on the hand paths reproduced 
based on the torque-change minimization criterion $\left(\omega_{1}=\omega_{2}=\omega_{3}=0.0, \omega_{4}=1.0\right)$; (a) represents the hand paths calculated by changing the value of $R_{11}\left(-2.0<=R_{11}<=2.0\right)$ at $R_{22}=R_{33}=1.0$; (b) represents the hand paths calculated by changing the value of $R_{22}\left(-2.0<=R_{22}<=2.0\right)$ at $R_{I I}=$ $R_{33}=1.0$; (c) represents the hand paths calculated by changing the value of $R_{33}\left(-2.0<=R_{33}<=\right.$ $2.0)$ at $R_{1 I}=R_{22}=1.0$. The target was $\mathrm{T} 5$ in the leftward movements. The optimization was executed by substituting the values of the state variables $\boldsymbol{x}_{0}$ and $\boldsymbol{x}_{t f}$ into the boundary conditions of Eq. (9) and solving the two-point boundary-value problem of Eq. (8) based on a Newton-like method. The first three components $\left(\theta_{1}, \theta_{2}, \theta_{3}\right)$ of the state variables $\boldsymbol{x}_{0}$ and $\boldsymbol{x}_{t f}$ were derived from one of the measured paths, and the other six components were fixed to zero as the arm was in a state of rest at the start and final positions. The gain values $k a, k v$, and $k p$ of the feedback controller were set to 0.147 (300 times as large as the moment of inertia of the hand), 0.0 , and 0.0 , respectively, according to our pre-calculation. All the diagonal component values of the weight matrix $\boldsymbol{Q}$ were set to $10^{7}$, and the movement duration was $t_{f}=922 \mathrm{msec}$. From Fig. 5 the following results were obtained: (1) when $R_{22}=$ $R_{33}=1.0$ (Fig. 5(a)), the hand path became a more concave curve toward the subject as the value of $R_{l l}$ increased in the positive range and became a more convex curve as the value of $R_{l l}$ decreased in the negative range; (2) when $R_{I I}=R_{33}=1.0$ (Fig. 5(b)), the hand path curved more concavely as the value of $R_{22}$ came close to zero in the positive range and curved more convexly as the value of $R_{22}$ came close to zero in the negative range; (3) when $R_{11}=R_{22}=1.0$ (Fig.5(c)), the hand path hardly changed regardless of the value of $R_{33}$. The results (1) and (2) will be because the optimal inputs (torque-changes) to the shoulder and elbow joints are influenced by the values of $R_{I I}$ and $R_{22}$, changing the angles of the both joints. The result (3) will be because the hand-joint's freezing-like mechanism (feedback controller) cancels the effect of changing $R_{33}$ on the hand joint. Therefore, the torque-change minimization criterion will make it possible to reproduce the measured trajectories if the values of the weight $\boldsymbol{R}$ are selected appropriately.

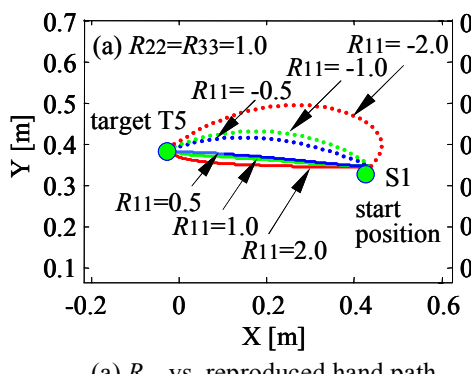

(a) $R_{11}$ vs. reproduced hand path

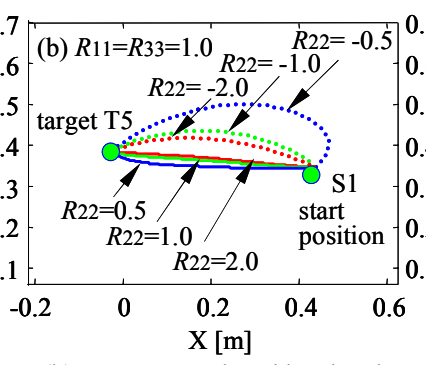

(b) $R_{22}$ vs. reproduced hand path

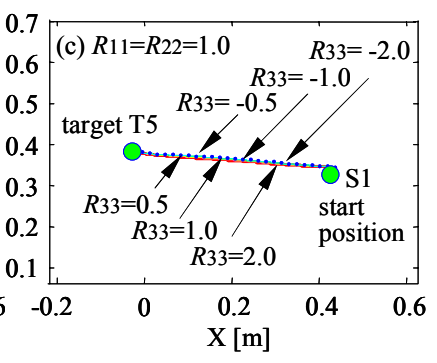

(c) $R_{33}$ vs. reproduced hand path.

Fig.5 Influence of the weight $\boldsymbol{R}$ on the reproduced hand path based on the torque-change minimization (from S1 to T5)

Figure 6 shows the comparison between the trajectories (dotted lines) measured experimentally (subject T.S.) and the trajectories (solid lines) reproduced based on the torque-change minimization criterion; (a) represents the hand paths for the leftward movements from $\mathrm{S} 1$ to the three targets (T4, T5, T6) and for the rightward movements from S2 to the two targets (T7, T8); (b) and (c) represent the shoulder-, elbow-, and hand-joint angle profiles and the tangential velocity profiles of the hand point for one of the measured leftward movements from S1 to T6. The diagonal component values of the input weight matrix $\boldsymbol{R}$ were searched so that the reproduced and measured trajectories agreed as well as possible. Actually, $R_{I I}=0.5, R_{22}=-1.0, R_{33}=1.5$ for Target T4; $R_{11}=1.1, R_{22}=-1.2, R_{33}=-1.0$ for Target T5; $R_{1 I}=0.2, R_{22}=-1.5, R_{33}=2.5$ for Target T6; $R_{11}=0.2, R_{22}=-0.2, R_{33}=1.5$ for Target $\mathrm{T} 7 ; R_{11}=0.5, R_{22}=-1.0, R_{33}=1.5$ for Target $\mathrm{T} 8$. The other calculation conditions were the same as those in Fig.5. The movement durations were $t_{f}=820,922,1125,996,956 \mathrm{msec}$ for T4, T5, T6, T7, and T8, respectively. From Fig. 6, the following results were obtained: 

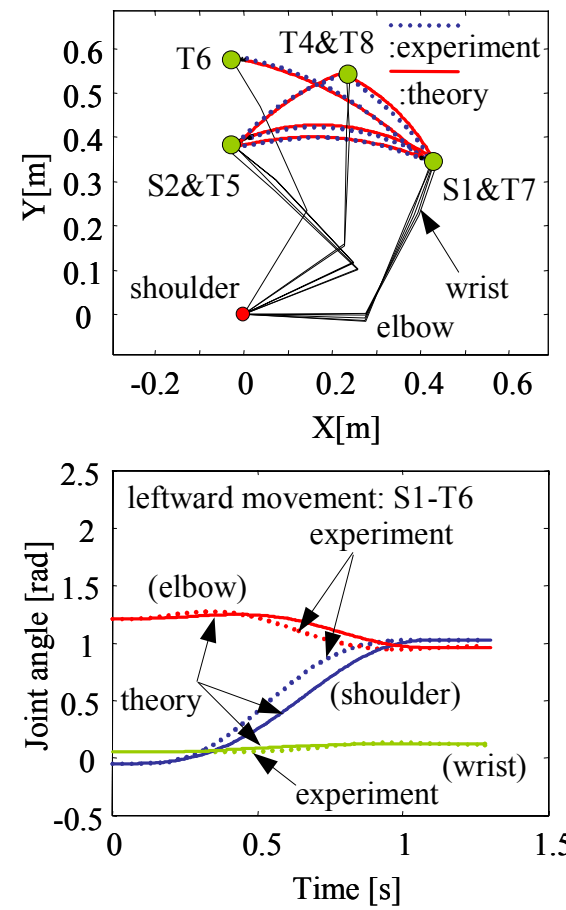

(b) Joint angle profiles for target $\mathrm{T} 6$ (a) Paths for the five target positions.

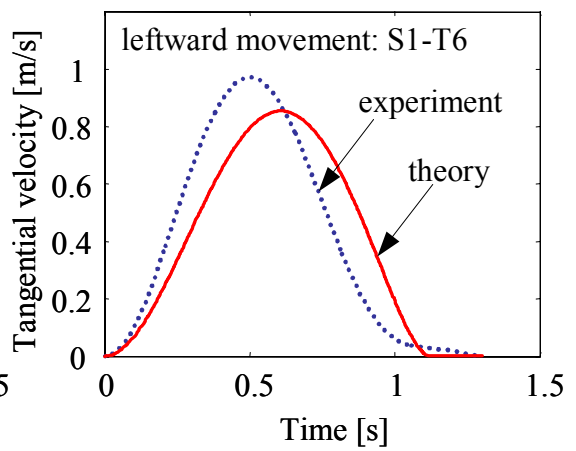

(c) Tangential velocity profile for target $\mathrm{T} 6$

Fig.6 Optimal trajectories based on the torque-change minimization criterion

(1) all the hand paths agreed well with the measured ones; (2) all the joint angle profiles including the hand joint agreed relatively well with the measured ones; (3) the hand-point's tangential velocity profile was successfully reproduced as a single-peaked and bell-shaped curve in the same way as the measured one. Moreover, additional calculations confirmed that the measured trajectories can never be reproduced without the feedback controller ( $k a=k v=k p=0.0$ ), because the model's hand joint is moved more than necessary under this condition, and that the excessive movement of the model's hand joint can never be suppressed by changing the model's weight values $\left(\omega_{4}\right.$ and $\left.\boldsymbol{R}\right)$. This implies that the hand-joint's feedback mechanism is indispensable for reproducing reaching movement characteristics of human three-joint arms with hand joints. Therefore, the above results mean that the torque-change minimization criterion makes it possible to reproduce human three-joint arm's reaching movement characteristics and that the hand-joint's feedback controller functions appropriately as a model of the human hand-joint's freezing-like mechanism. In order to obtain the above results, however, it was essential to adjust the weight matrix $\boldsymbol{R}$ in advance to the optimal values for each target. This can be a defect of the torque-change minimization criterion. That is because the adjustment itself is a kind of optimization and a hard task.

\subsection{Trajectory reproduction based on energy minimization criterion}

Figure 7 shows the hand paths reproduced by minimizing the criterion function of Eq. (4), i.e., the energy term plus the torque-change term, as a parameter of each of three kinds of weights $\left(\omega_{1}, \omega_{2}, \omega_{3}\right)$; (a), (b), and (c) represent the hand paths reproduced by minimizing the moment power of joints $\left(\omega_{1}\right.$ : parameter, $\left.\omega_{2}=\omega_{3}=0\right)$ for one measured leftward movement from S1 to T6, the energy for sustaining the torque of joints $\left(\omega_{2}\right.$ : parameter, $\left.\omega_{1}=\omega_{3}=0\right)$ for one measured leftward movement from S1 to T5, and the energy consumed by viscous resistance $\left(\omega_{3}\right.$ : parameter, $\left.\omega_{1}=\omega_{2}=0\right)$ for one measured rightward movement from S2 to T8, respectively; (1) and (2) are for subjects T.S. and K.T., respectively. For comparison, the experimentally measured hand paths (dotted fat lines) are also added to Fig. 7. The 
movement durations for Fig. 7 (a), (b), and (c) were $t_{f}=1125,922,956 \mathrm{msec}$, respectively, for subject T.S. and $t_{f}=825,808,739 \mathrm{msec}$, respectively, for subject K.T.; all the diagonal component values of the weight matrices $\boldsymbol{Q}$ and $\boldsymbol{R}$ were fixed to $10^{7}$ and 1.0, respectively; the weight value $\omega_{4}$ was fixed to 0.01 . In this optimization, since the weight $\omega_{4}$ of the input term (torque-change term) in the criterion function was set to a much smaller value than the other weights $\left(\omega_{1}, \omega_{2}, \omega_{3}\right)$, it should largely weaken the effect of decreasing the torquechange: so minimizing the criterion function of Eq. (4) can be practically regarded as minimizing only the energy term. From Fig. 7 the following results were obtained; (1) as for minimizing the moment power of joints (Fig.7(a)), the reproduced hand path changed gradually from a concave curve to a convex one with an increase in $\omega_{l}$ and agreed nearly with the measured path at a value of $\omega_{1} ;(2)$ as for minimizing the energy for sustaining the torque of joints (Fig.7(b)), the reproduced hand path maintained almost the same concave curve regardless of the value of $\omega_{2}$ and never agreed with the measured path; (3) as for minimizing the energy consumed by viscous resistance (Fig.7(c)), the reproduced hand path changed gradually from a concave curve to a convex one with an increase in the value of $\omega_{3}$ and agreed nearly with the measured path at a value of $\omega_{3}$; (4) the above results were independent of the start and target positions and the subjects. The above results make us expect a possibility that, if the value of $\omega_{1}$ or $\omega_{3}$ is appropriately selected once for each subject, the minimization of the moment power of joints or that of the energy consumed by viscous resistance will make it possible to reproduce the measured hand paths regardless of the start and target positions and subjects.
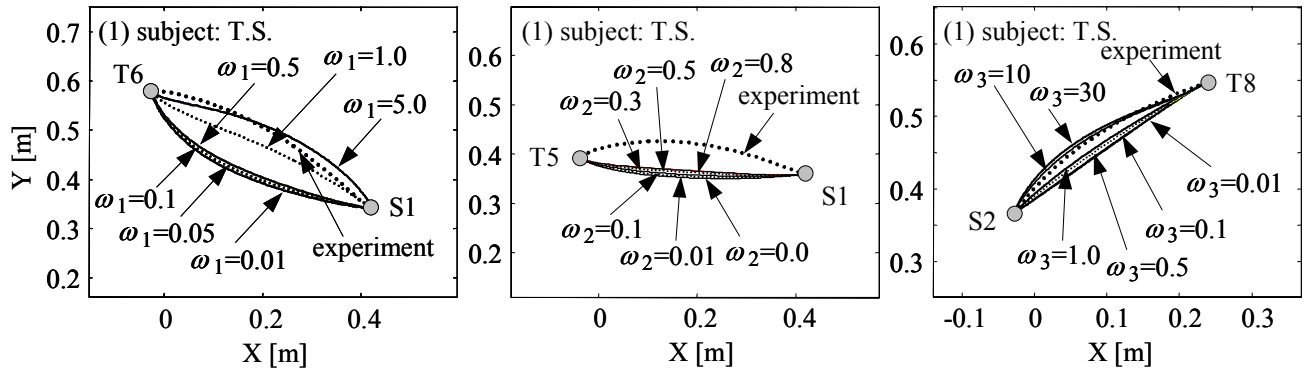

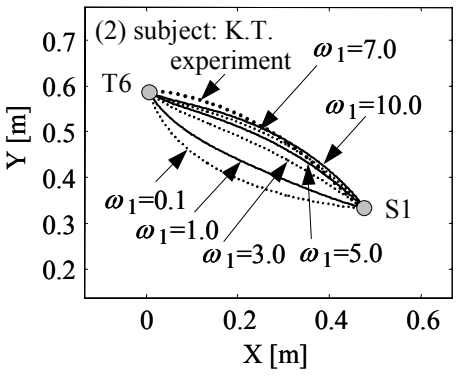

(a) joint moment power

(S1-T6)

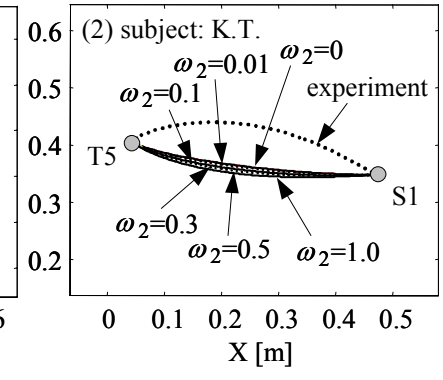

(b) torque-sustaining energy

(S1-T5)

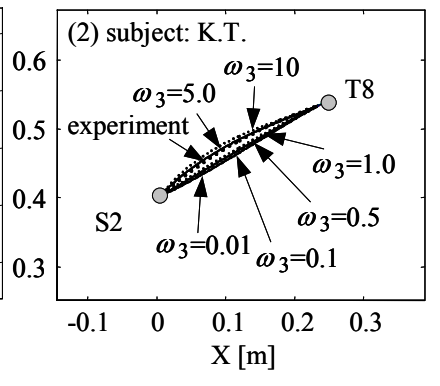

(c) energy consumed by viscous resistance (S2-T8)

Fig.7 Influence of the energy weights on the reproduced hand path based on the energy minimization

Figure 8 shows the comparison between the trajectories (dotted lines) measured experimentally and the trajectories (solid lines) reproduced by minimizing the moment power of joints $\left(\omega_{2}=\omega_{3}=0\right)$ at a constant weight value of $\omega_{1}$. This calculation was executed to verify whether the hand paths predicted at the constant value of $\omega_{1}$ agree well with the measured ones regardless of the start and target positions and the subjects, in other words, whether the defect of the torque-change minimization criterion can be overcome. Fig.8 (a) represents the hand paths for three kinds of leftward movements from S1 to the three targets (T4, T5, T6) and for two kinds of rightward movements from S2 to the two targets (T7, T8); Fig.8 (b) and (c) represent the shoulder-, elbow-, and hand-joint angle profiles and the 
tangential velocity profiles of the hand point for one of the measured leftward movements from S1 to T6; (1) and (2) are for subjects T.S. and K.T., respectively. The movement durations were $t_{f}=820,922,1125,996,956 \mathrm{msec}$ for T4, T5, T6, T7, and T8, respectively, for subject T.S. and $t_{f}=721,808,825,829,739 \mathrm{msec}$ for subject K.T.; the value of $\omega_{1}$ was 3.0 and 5.0 for subjects T.S. and K.T., respectively; the values of the weight matrices $\boldsymbol{Q}$ and $\boldsymbol{R}$ and the weight value $\omega_{4}$ were the same as in Fig.7. From Fig. 8, the following results were obtained: (1) all the reproduced hand paths agreed well with the measured ones regardless of the start and target positions in spite of the constant value of $\omega_{I}$; (2) the reproduced joint angle profiles including the hand joint agreed relatively well with the measured ones in almost the same way as in the case of the torque-change minimization criterion (Fig. 6); (3) the hand-point's tangential velocity profile was successfully reproduced as a single-peaked and bell-shaped curve in the same way as the measured one; (4) the above results were independent of the subjects. Moreover, we also verified whether the minimization of the energy consumed by viscous resistance $\left(\omega_{1}=\omega_{2}=0\right)$ can reproduce the measured trajectories at the constant value of $\omega_{3}$ regardless of the start and target positions and the subjects, and confirmed that the derived results are the same as the above four results. The above results mean that the minimization of the moment power of joints and that of the energy consumed by viscous resistance have the ability to reproduce measured trajectories regardless of start and target positions and subjects: that is, the above
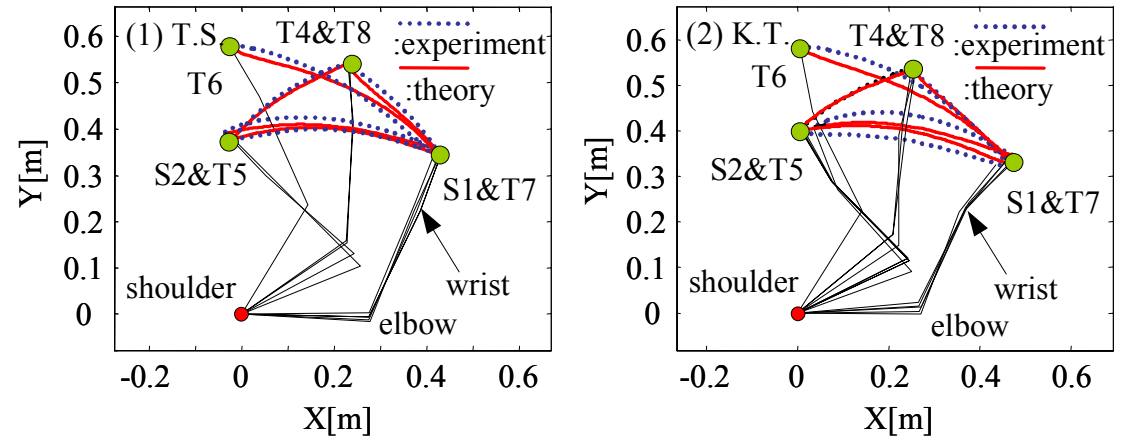

(a) Hand paths for the five target positions.
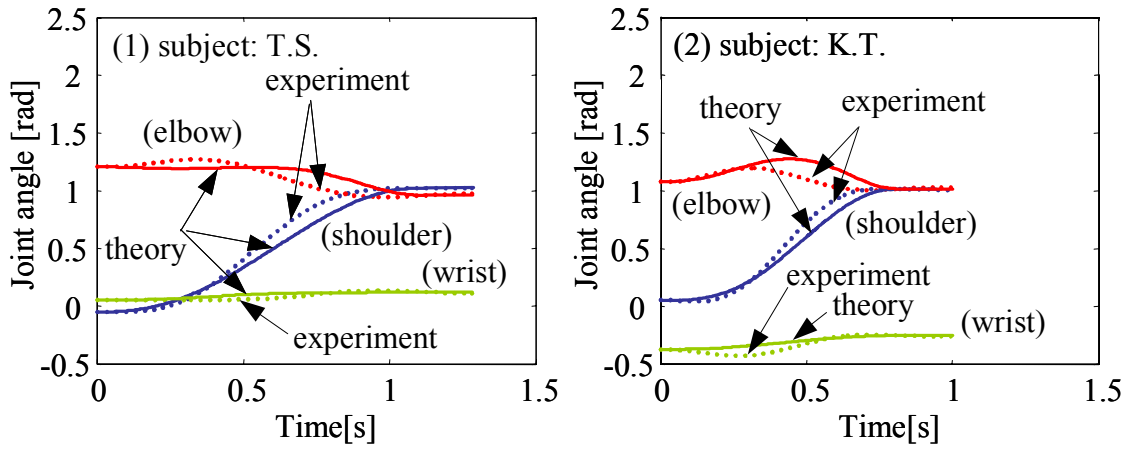

(b) Joint angle profiles from S1 to T6
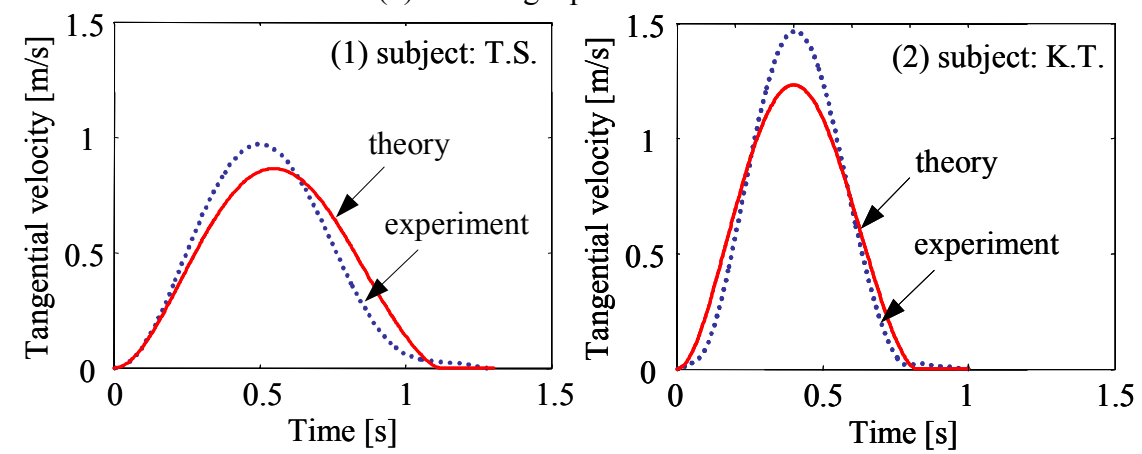

(c) Tangential velocity profiles from S1 to T6

Fig.8 Optimal trajectories based on the minimization of the moment power of joints 
two kinds of energy minimization criteria can overcome the defect of the torque-change minimization criterion.

Figure 9 shows the comparison between the joint angle profiles (dotted lines) measured experimentally and the joint angle profiles (solid lines) reproduced by minimizing the energy consumed by viscous resistance $\left(\omega_{1}=\omega_{2}=0\right)$. This calculation was executed to verify whether the reproduced hand-joint profiles agree well with the measured ones regardless of the start and target positions and subjects, in other words, how the hand-joint's freezing-like characteristics can be reproduced. Fig.9 (a) and (b) are for two kinds of leftward movements from S1 to T4 and T5, respectively; (c) is for one rightward movement from S2 to T8; (1) and (2) are for subjects T.S. and K.T., respectively. The movement durations were $t_{f}=820$, $922,956 \mathrm{msec}$ for T4, T5, and T8, respectively, for subject T.S. and $t_{f}=721,808,739 \mathrm{msec}$ for subject K.T.; the value of $\omega_{3}$ was 3.0 and 5.0 for subjects T.S. and K.T., respectively; the values of the weight matrices $\boldsymbol{Q}$ and $\boldsymbol{R}$ and the weight value $\omega_{4}$ were the same as in Figs.7 and 8. From Fig. 9, the following results were obtained: (1) the reproduced shoulder- and elbow- joint angles changed definitely during the movement duration depending on the start and target positions; (2) although the reproduced hand-joint angle hardly changed over the movement duration, it was not necessarily constant but changed a little bit depending on the start and target positions; (3) the transition characteristics for the reproduced joint angles agreed well with those for the measured ones. Moreover, additional calculations also confirmed that the proposed model can never reproduce the measured joint angle profiles without its hand-joint's feedback controller ( $k a=k v=k p=0.0$ ), because its hand joint is moved more than necessary under this condition, and that the excessive movement of the model's hand joint can never be suppressed by changing the model's weight values $\left(\omega_{1}, \omega_{3}, \omega_{4}\right.$, and $\boldsymbol{R})$. The above results mean that the feedback controller installed in the model's hand joint can not only make the hand joint almost fixed, but also make it flexibly changed in case of need: in other words, the feedback controller can be an appropriate model for the hand-joint's freezing-like mechanism.

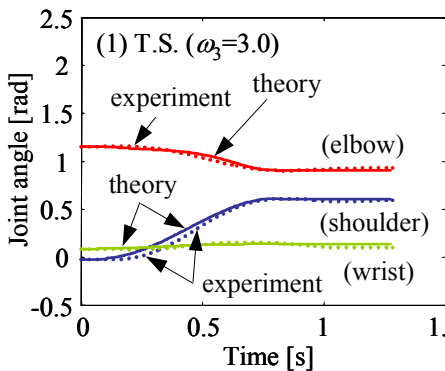

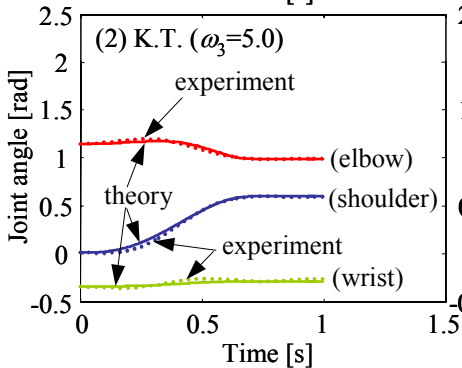

(a) leftward movements (S1-T4)
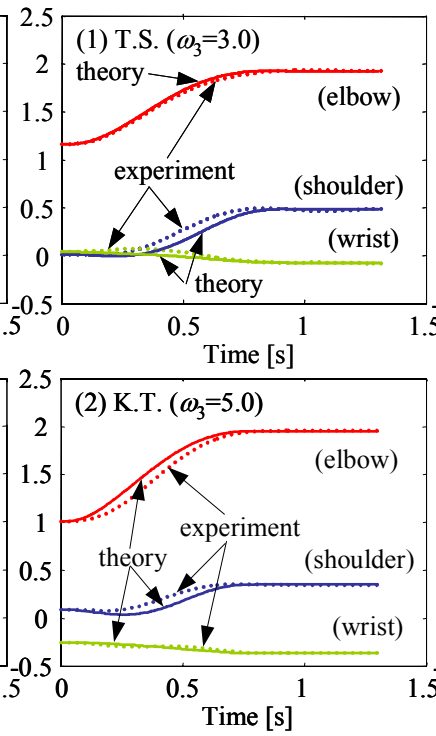

(b) leftward movements (S1-T5)
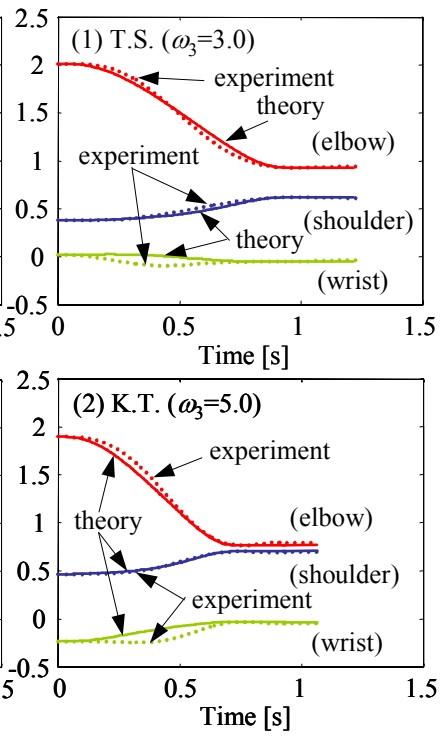

(c) rightward movements (S2-T8)

Fig.9 Optimal joint angle profiles reproduced by minimizing the energy consumed by viscous resistance

\section{Discussion}

\subsection{Model for hand-joint's freezing-like mechanism}

Fig. 3 clarified that the hand-joint angle hardly changes during human three-joint arm's 
reaching movements regardless of the start and target positions and the subjects. That is because the freezing-like mechanism functions to make the hand joint hardly move. Judging from the function of hands, hands can fundamentally be regarded as an end-effector; so, it would hardly be necessary to move our hand joints in the case of three-joint arm's simple reaching movements without any constraints. However, Fig. 3 also clarified that it is possible to change the hand-joint angle a little bit depending on the start and target positions even if the hand-joint's freezing-like mechanism prevents the hand joint from moving. Moreover, situations will arise where we have to move our hand joints intentionally during reaching movements as in the case of holding a cup's handle with fingertips at the final point or avoiding obstacles on the way to the target. Therefore, if the proposed model can be a general and plausible model for the human three-joint arm's control mechanism, it should be able to reproduce the above two kinds of freezing-like characteristics: one is to hardly change the hand-joint angle during reaching movements; the other is to change it flexibly in case of need.

In this paper, from the above point of view, the hand-joint's freezing-like mechanism was modeled as a feedback controller (Eq. (1)) that produces the feedback torque $\tau_{3} *$ in the direction opposite to the hand-joint motion. Actually, according to our pre-calculation, an acceleration-based feedback controller was adopted: the gain values $k a, k v$, and $k p$ of the feedback controller were set to $0.147,0.0$, and 0.0 , respectively. Though a velocity-based feedback controller $(k a=k p=0.0)$ and an angle-based feedback controller $(k a=k v=0.0)$ could also prevent the model's hand joint from moving, they tended to make the model's hand joint oscillate; that is why these two feedback controllers were not adopted as a model of the hand-joint's freezing-like mechanism. Incorporating the acceleration-based feedback controller into the model's hand joint is equivalent to increasing the value of the $(3,3)$ component $M_{33}$ of the inertia matrix $\boldsymbol{M}$, in other words, the mass $m_{3}$ of the hand portion. Therefore, if the $(3,3)$ component value, i.e., the mass of the hand portion becomes larger, the hand joint will be definitely more difficult to move; however, the hand joint cannot completely be fixed. That will be why the three-joint arm model with the freezing-like mechanism in its hand-joint can change its hand joint a little depending on the start and target positions in case of need, as well as hardly changing its hand joint over reaching movements. Therefore, the installed feedback controller can be an effective and general model for the human hand-joint's freezing-like mechanism.

\subsection{Comparison with conventional optimal control models}

As shown in Figs. 5 and 6, the torque-change minimization $\left(\omega_{1}=\omega_{2}=\omega_{3}=0.0, \omega_{4}=1.0\right)$ did not make it possible to simulate human three-joint arm's reaching movements, and in order to make it possible to do so, it was essential to adjust the weight matrix $\boldsymbol{R}$ in advance to the optimal values for each target: the minimum torque-change model is not always satisfactory as a model for the human three-joint arm control system. In contrast, as seen from Figs. 7, 8, and 9, the minimization of the moment power of joints or that of the energy consumed by viscous resistance made it possible to simulate human three-joint arm's reaching movements at the constant weight value of $\omega_{1}$ or $\omega_{3}$ regardless of the start and target positions. The above results suggest that the moment power of joints or the energy consumed by viscous resistance can be involved in the trajectory planning for human three-joint arm's reaching movements, though the torque sustaining energy and the torquechange cannot be done. On the other hand, some research pointed out that the two-joint (shoulder and elbow joints) arm's minimum torque-change model could simulate human two-joint arm's reaching movements without adjusting the weight matrix $\boldsymbol{R}^{(4)(5)}$ and that the two-joint arm's minimum energy model could simulate them by minimizing the torquesustaining energy and the energy consumed by viscous resistance ${ }^{(6)}$. These results suggest that the torque-change, the torque-sustaining energy, and the energy consumed by viscous 
resistance can be involved in the trajectory planning for human two-joint arm's reaching movements: this is different from our suggestion from the proposed model. The difference in the suggestion between the conventional minimum torque-change model and the proposed model can be thought to be caused by the number of joints and the existence of the freezing-like mechanism. Meanwhile, the difference in the suggestion between the conventional energy minimization model and the proposed model can be thought to be caused by the existence of the torque-change term in the criterion function (Eq. (4)) as well as the number of joints and the existence of the freezing-like mechanism. There exists no torque-change term in the conventional energy minimization model ${ }^{(6)}$. In the proposed model, however, since the weight value $\left(\omega_{4}=0.01\right)$ of the torque-change term is considerably smaller than the other weight values $\left(\omega_{1}=\omega_{3}=3\right.$ or 5$)$ of the energy term, the criterion function can be expected to work as an energy minimization even if the torque-change term exists. For confirmation, we examined what hand trajectories are reproduced by minimizing the following criterion function with no torque-change term:

$$
J=\left\{\boldsymbol{x}\left(t_{f}\right)-\boldsymbol{x}_{t f}\right\}^{\mathrm{T}} \boldsymbol{Q}\left\{\boldsymbol{x}\left(t_{f}\right)-\boldsymbol{x}_{t f}\right\}+\int_{0}^{t_{f}}\left(\omega_{1} \tau^{\mathrm{T}} \dot{\boldsymbol{\theta}}+\omega_{2} \tau^{\mathrm{T}} \tau+\omega_{3} \dot{\boldsymbol{\theta}}^{\mathrm{T}} \boldsymbol{D} \dot{\boldsymbol{\theta}}\right) d t
$$

As a result, we confirmed again that the minimization of the torque-sustaining energy does not make it possible to simulate the human three-joint arm's reaching movements. This suggests that the existence of the torque-change term cannot be a factor that causes the difference between the conventional energy minimization model and the proposed model. In addition, there can also be a possibility that the differences between the conventional two-joint models and the proposed three-joint model may be caused by the difference in experimental conditions such as experimental apparatus or subjects. Therefore, it will also be necessary to verify the above discussion by measuring two-joint arm's and three-joint arm's reaching movements under the same conditions, and this will be our future work.

\section{Conclusion}

We formulated a new human three-joint arm's optimal control model characterized by a freezing-like mechanism in its hand joint and three kinds of energy costs in its criterion function. The proposed model was applied to simulating human three-joint arm's reaching movements, and the following results were obtained; (1) the torque-change minimization required the optimal adjustment of the model's weight values $\boldsymbol{R}$ for each target to reproduce the measured trajectories: this is a defect of this criterion; (2) the minimization of moment power of joints or that of energy consumed by viscous resistance made it possible to reproduce the measured trajectories using the constant value of the weight $\omega_{1}$ or $\omega_{3}$ regardless of the start and target positions; (3) the proposed model successfully reproduced the measured hand-joint's freezing-like characteristics. These suggest that the proposed model can be effective as a general and plausible model of the human three-joint arm control system and that the moment power of joints and the energy consumed by viscous resistance can be involved in the trajectory planning for human three-joint arm's reaching movements.

\section{References}

(1) Morasso, P., Spatial Control of Arm Movements, Exp. Brain Research, Vol. 42 (1981), pp. 223-227.

(2) Abend, W., Bizzi, E., and Morasso, P., Human Arm Trajectory Formation, Brain, Vol. 105 (1982), pp. 331-348.

(3) Flash, T. and Hogan, N., The Coordination of Arm Movements: An Experimentally Confirmed Mathematical Model, J. Neuroscience, Vol. 5 (1985), pp. 1688-1703.

(4) Uno, Y., Kawato, M., and Suzuki, R., Formation and Control of Optimal Trajectory in Human Multijoint Arm Movement - Minimum Torque-Change Model, Biol. Cybern., Vol. 
61 (1989), pp. 89-101.

(5) Nakano, E., Imamizu, H., Osu, R., Uno, Y., Gomi, H., Yoshioka, T., and Kawato, M., Quantitative Examination of Internal Representations for Arm Trajectory Planning: Minimum Commanded Torque Change Model, J. Neurophysiol., Vol. 81 (1999), pp. 2140-2155.

(6) Kashima, T. and Isurugi, Y., Trajectory Formation in Human Arm Movement, Transaction of SICE, Vol. 31, No. 9 (1995), pp. 1416-1422.

(7) Kashima, T. and Isurugi, Y., Human Arm Trajectory Formation, Transaction of SICE, Vol. 34, No. 10 (1998), pp. 1440-1447.

(8) Harris, C.M. and Wolpert, D.M., Signal- Dependent Noise Determines Motor Planning, Nature, Vol. 394, No. 20 (1998), pp. 780-784.

(9) Matsui, T., Honda, M., and Nakazawa, N., A New Optimal Control Model for Reproducing Human Arm's Two-Point Reaching Movements: A Modified Minimum Torque Change Model, Proceedings of the 2006 IEEE International Conference on Robotics and Biomimetics (2006), pp.1541-1546.

(10) Bernstein, N., The Co-ordination and Regulation of Movements, Pergamon Press, London (1967).

(11) Gatev, U., Role of Inhibition in the Development of Motor Co-ordination in Early Childhood, Developmental Medicine and Child Neurology, Vol. 14 (1972), pp. 336-341.

(12) Nielsen, J. and Kagamihara, Y., The Regulation of Disynaptic Reciprocal Ia Inhibition during Co-contraction of Antagonistic Muscles in Man, J. Physiology, Vol. 456 (1992), pp. 373-391. 